\title{
Análisis de las universidades colombianas de acuerdo con el ranking SCImago 2010-2012
}

\section{Analysis of the Colombian universities according to SCImago ranking 2010-2012}

\author{
Salim Mattar V, ${ }^{1 *}$ Ph.D, Marco González T, ${ }^{1}$ M.Sc, Luis Salgado A, ${ }^{1}$ Ing. \\ ${ }^{1}$ Universidad de Córdoba, Facultad de Medicina Veterinaria y Zootecnia. Departamento de Ciencias \\ Pecuarias. Montería, Colombia. Correspondencia: mattarsalim@hotmail.com
}

Recibido: Octubre de 2012; Aceptado: Enero de 2013.

\section{RESUMEN}

Objetivo. Analizar la clasificación de las universidades colombianas de acuerdo con el escalafón Iberoamericano SCImago Institutions Ranking (SIR) 2012. Materiales y métodos. Se realizó un análisis descriptivo que incluyó la productividad académica de cada universidad según SIR. También, se incluyeron las clasificaciones de 2010 y 2011 para analizar el progreso académico de las universidades. El análisis incluyó los siguientes indicadores: producción científica, colaboración internacional, calidad científica promedio, porcentaje de publicaciones en revistas del primer cuartil SJR y radio de excelencia. Resultados. Colombia ocupa el tercer lugar con 113 Universidades en Iberoamérica y el séptimo puesto con 7 universidades con más de 400 documentos. Sólo el $6.2 \%$ de las instituciones colombianas mantienen una producción científica competitiva frente al resto de las universidades Iberoamericanas. Las cinco primeras universidades colombianas con mayor productividad fueron: Nacional de Colombia, Antioquia, Andes, Valle y Javeriana. Las cinco primeras universidades del Caribe fueron: Norte, Cartagena, Córdoba, Magdalena y Sucre. Algunas universidades privadas como Antonio Nariño, el Bosque y la Escuela Colombiana de Ingenieria, a pesar de no estar dentro de los quince primeros puestos del pais, han hecho esfuerzos para mejorar los indicadores de calidad científica mediante publicaciones en revistas del primer cuartil y radio de excelencia. Conclusiones. Las clasificaciones que permiten comparar las IES a nivel mundial son importantes. Las IES de Colombia deben apostarle a la calidad usando al menos los cinco factores de SCImago. Aunque los rankings no obligan, ignorarlos significa autoaislamiento en un mundo cada día más globalizado.

Palabras clave: Categorización, educación, visibilidad (Fuente: CAB). 


\section{ABSTRACT}

Objective. To analyze the classification of Colombian universities according to the Latin-american and spanish ranking SCImago Institutions rankings (SIR) 2012. Materials and methods. A descriptive analysis was performed, which included academic productivity of each University according to SIR. 2010 and 2011 classifications were also included to discuss the academic progress of the universities. The analysis included the following indicators: scientific production, international collaboration, scientific quality, average percentage of publications in journals of the first quartile SJR and ratio of excellence. Results. Colombia ranks third place with 113 universities in Latin America and the seventh place with 7 universities with more than 400 documents. Only $6.2 \%$ of the Colombian institutions maintain a competitive scientific production compared to the rest of the Latin-American and spanish universities. The first five Colombian universities with higher productivity were: Nacional, Antioquia, Andes, Valle, and Javeriana. The first five universities of the Caribbean were: Norte, Cartagena, Córdoba, Magdalena and Sucre. Some private universities such as Antonio Nariño, El Bosque and the Colombian School of Engineering, despite not being within the first fifteen places of the country, they have made efforts to improve the indicators of scientific quality through publications in journals of the first quartile and radio excellence. Conclusions. The classifications that allow to compare the IES at the global level are important. The IES of Colombia should bet on quality using at least five factors SCImago ranking. Although the rankings are not mandatory, ignoring them means self-isolation in a world each day more globalized.

Key words: Education, ranking, visibility (Source: $C A B$ ).

\section{INTRODUCCIÓN}

La universidad moderna está concebida como el claustro para la construcción de la sociedad, tanto en su papel de educar como el de difundir el conocimiento y por lo tanto, debe ser el eje principal del desarrollo humano sostenible que impulse la transformación de la sociedad. Es la carta de presentación de un país, que incluye la política de la educación superior y debe propender por un profundo desarrollo humano que impacte en la sociedad, promoviendo la rentabilidad social sostenible en el tiempo.

La universidad no puede ser ajena a la dinámica mundial. En ese sentido, el mundo académico es cada día más exigente con sus profesores y estudiantes. Para ello se han implementado modelos destinados a medir la calidad de la educación, que mediante diversas variables objetivas, intentan calificar a las instituciones de educación superior. Aunque el fenómeno de la calidad es complejo debido a la diferencia social mundial y a las condiciones individuales de trabajo de cada universidad $(1,2)$, existen variables comunes que permiten acercarlas con una medición homogénea.

Las mediciones o rankings (en inglés) son motivo de debate en los claustros universitarios debido a las metodologías, que al parecer de algunos, no toman en cuenta las diferencias existentes entre las universidades del mundo. No obstante, estas clasificaciones se han convertido en una herramienta adecuada que utilizan las universidades para aumentar la visibilidad y la competitividad $(2,3)$. Los rankings son sistemas que se utilizan para clasificar listados de universidades, programas, revistas, utilizando diferentes metodologías de evaluación, lo que ofrece una clara visión de la calidad de la educación superior de un país, su ubicación y la relación con su entorno y el mundo $(4,5)$.

Entre los rankings más destacados a nivel mundial se encuentra el Academic Ranking of World Universities, elaborado por la Universidad Jiao Tong de Shanghai (China), el cual clasifica las primeras 500 universidades del mundo, de acuerdo con los siguientes criterios de evaluación: calidad de la educación, calidad del personal académico, producción investigativa, tamaño de la institución (6). The World University Rankings, elaborado por el Times Higher Education, que incluye las 400 mejores universidades del mundo las califica de acuerdo a 13 indicadores divididos en 5 grandes categorías: i) Docencia: entorno de aprendizaje; ii) Investigación: cantidad, ingresos, reputación; iii) Referencias: impacto de la investigación; iv) Ingresos empresariales: innovación y v) Aspectos internacionales: personal, estudiantes e investigación (7). El Webometrics Ranking tiene como objetivo principal apoyar las iniciativas de acceso abierto y promover el acceso global al conocimiento académico producido por universidades en todo el mundo (8). Finalmente, está el proyecto SCImago "SCImago Instituciones Rankings (SIR)", el cual permite la clasificación de universidades Iberoamericanas, teniendo 
en cuenta los siguientes factores: producción científica, colaboración internacional, calidad científica promedio, porcentaje de publicaciones en revistas del primer cuartil en SCImago Journal \& Country Rank (SJR) que es un portal que incluye los indicadores científicos de país desarrollados a partir de la información contenida en la base de datos Scopus ${ }^{\circledR}$ (9) y por último el radio de excelencia (10).

Por tal motivo, los rankings de las universidades se han convertido en un elemento indispensable y "neutral" para medir el nivel de impacto que tienen a nivel del país, de la región y del mundo. Bajo la perspectiva de la calidad de la educación y de la divulgación del conocimiento, estos rankings facilitan a las universidades la posibilidad de diseñar estrategias que les permitan competir de una forma justa y transparente en busca de los primeros lugares de los mismos.

El objetivo del presente estudio fue realizar un análisis descriptivo del estado de las universidades colombianas siguiendo la clasificación propuesta por la última actualización del Ranking Iberoamericano SCImago SIR 2012.

\section{MATERIALES Y MÉTODOS}

Muestra. Se tomaron las primeras 50 universidades Colombianas del Ranking Iberoamericano Scimago 2012 (10), lo que equivale al $3.7 \%$ del total de Universidades iberoamericanas dentro del ranking. Para analizar y comparar el progreso académico de las universidades en el tiempo, también se incluyeron las clasificaciones de SCImago de 2010 y 2011 (10).

Análisis de los datos. Los registros procedentes del ranking Iberoamericano SIR 2012 se integraron a una base de datos y mediante una hoja de cálculo de Ms Excel ${ }^{\circledR}$ se elaboraron tablas y figuras. Se emplearon indicadores bibliométricos cuantitativos basados en el análisis de la información contenida en dicho ranking y se discriminaron por las variables o indicadores utilizadas por Scimago:

Producción Científica (PC). Es la producción de la institución medida en número de publicaciones en revistas científicas. PC ofrece una idea general del tamaño de una institución. En publicaciones con coautoría, se asigna un punto a cada una de las instituciones participantes.

Colaboración Internacional (CI). Ratio de publicaciones científicas de una institución que han sido elaboradas conjuntamente con otros países. Los valores se calculan analizando las publicaciones de una institución cuya filiación incluye direcciones pertenecientes a más de un país.

Calidad Científica Promedio (CCP). Impacto científico de una institución después de eliminar la influencia del tamaño y el perfil temático de la institución. EI CCP permite comparar instituciones de diferentes tamaños y con distintos perfiles de investigación. Una puntuación de 0.8 significa que una institución es citada un $20 \%$ menos que la media mundial. Un valor de 1.3 indica que la institución es citada un $30 \%$ más que la media mundial.

Porcentaje de Publicaciones en Revistas del Primer Cuartil SJR (Q1). Indica el porcentaje de publicaciones que una institución ha publicado en revistas incluidas en el primer cuartil (25\%) ordenadas por el indicador SJR. El indicador SJR mide la influencia o prestigio científico de las revistas mediante el análisis de la cantidad y la procedencia de las citas que recibe una revista científica. Su uso se ha extendido a través del portal SCImago Journal \& Country Rank y es utilizado por Elsevier en su índice de citas Scopus (9).

Ratio de Excelencia (ER). Indica el porcentaje de la producción de una institución que se encuentra incluida en el $10 \%$ de los trabajos más citados del mundo en sus respectivos campos científicos. Es un indicador de la producción científica de alta calidad que produce una institución.

\section{RESULTADOS}

Al analizar a Colombia dentro del Ranking SIR 2012, se puede observar que ocupa el tercer lugar con 113 universidades en Iberoamérica (Figura 1A) y el séptimo puesto con 7 universidades con más de 400 documentos (Figura 1B), en ese sentido, sólo el $6.2 \%$ de las instituciones colombianas dentro del SIR 2012, mantienen una producción científica competitiva frente al resto de las universidades Iberoamericanas.

Las 50 primeras universidades de Colombia de un total de 113 en el listado del Ranking Iberoamericano en el período 2010-2012, se muestran en la tabla 1. De las 113 universidades colombianas, el $44 \%$ son públicas y el $56 \%$ privadas. En la tabla 1 se puede ver como algunas universidades se han mantenido en sus puestos durante estos tres años, como la Universidad Nacional de Colombia y la Universidad de Antioquia; otras han perdido o escalado posiciones tanto a nivel nacional como internacional. 


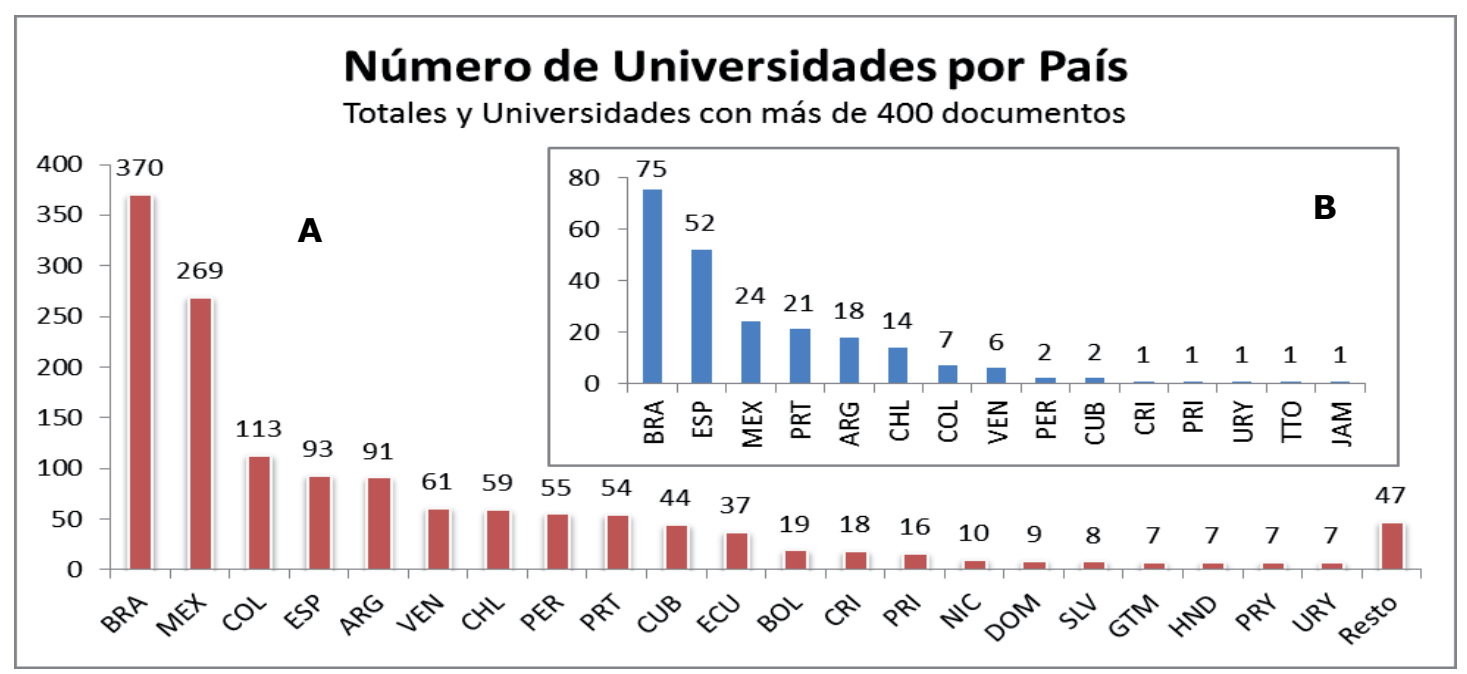

A. Columnas rojas se observa el número de universidades por país dentro del ranking. B. Columnas azules universidades con más de 400 documentos publicados.

Figura 1. Clasificación de países dentro del Ranking Iberoamericano 2012, según SCImago (10).

Tabla 1. Clasificación de las primeras 50 Universidades colombianas, 2010-2012.

\begin{tabular}{|c|c|c|c|c|c|c|c|c|c|}
\hline \multirow{2}{*}{ Universidad } & \multicolumn{3}{|c|}{2012} & \multicolumn{3}{|c|}{2011} & \multicolumn{3}{|c|}{2010} \\
\hline & COL & IBE & LAC & COL & IBE & LAC & COL & IBE & LAC \\
\hline Universidad Nacional de Colombia & 1 & 47 & 22 & 1 & 56 & 25 & 1 & 67 & 29 \\
\hline Universidad de Antioquia & 2 & 78 & 37 & 2 & 81 & 37 & 2 & 87 & 37 \\
\hline Universidad de los Andes, Colombia & 3 & 97 & 46 & 3 & 108 & 56 & 4 & 121 & 68 \\
\hline Universidad del Valle & 4 & 117 & 63 & 4 & 119 & 65 & 3 & 118 & 65 \\
\hline Pontificia Universidad Javeriana & 5 & 144 & 85 & 5 & 153 & 92 & 5 & 154 & 92 \\
\hline Universidad Industrial de Santander & 6 & 150 & 91 & 6 & 162 & 101 & 6 & 158 & 96 \\
\hline Universidad del Rosario & 7 & 205 & 136 & 7 & 219 & 148 & 7 & 226 & 152 \\
\hline Universidad Pontificia Bolivariana & 8 & 244 & 168 & 8 & 244 & 168 & 8 & 236 & 161 \\
\hline Universidad del Norte & 9 & 257 & 180 & 10 & 318 & 233 & 14 & 307 & 221 \\
\hline Universidad del Cauca & 10 & 275 & 197 & 9 & 298 & 216 & 9 & 259 & 178 \\
\hline Universidad Tecnológica de Pereira & 11 & 289 & 210 & 12 & 333 & 247 & 12 & 304 & 218 \\
\hline Universidad de Caldas & 12 & 299 & 219 & 11 & 328 & 242 & 10 & 296 & 210 \\
\hline Universidad de Cartagena & 13 & 312 & 230 & 13 & 337 & 251 & 13 & 306 & 220 \\
\hline Universidad EAFIT & 14 & 318 & 235 & 15 & 343 & 257 & 11 & 298 & 212 \\
\hline Universidad Distrital Francisco José de Caldas & 15 & 334 & 248 & 18 & 368 & 280 & 20 & 346 & 254 \\
\hline Universidad del Quindío & 16 & 339 & 253 & 20 & 383 & 293 & 18 & 333 & 244 \\
\hline Universidad de Cordoba & 17 & 341 & 255 & 17 & 366 & 278 & 21 & 355 & 262 \\
\hline Universidad CES & 18 & 347 & 261 & 14 & 339 & 253 & 15 & 315 & 228 \\
\hline Universidad de La Sabana & 19 & 355 & 268 & 21 & 384 & 294 & 22 & 356 & 263 \\
\hline Universidad El Bosque & 20 & 363 & 275 & 16 & 358 & 272 & 17 & 330 & 241 \\
\hline Universidad del Tolima & 21 & 374 & 286 & 23 & 429 & 334 & 24 & 361 & 268 \\
\hline Universidad Pedagógica y Tecnológica de Colombia & 22 & 385 & 297 & 22 & 405 & 312 & 23 & 360 & 267 \\
\hline Universidad de Pamplona & 23 & 389 & 301 & 19 & 372 & 284 & 16 & 323 & 235 \\
\hline Universidad Autónoma de Occidente & 24 & 404 & 311 & 29 & 482 & 385 & 29 & 388 & 292 \\
\hline Universidad de Bogotá Jorge Tadeo Lozano & 25 & 425 & 330 & 26 & 436 & 341 & 31 & 393 & 297 \\
\hline Universidad Militar Nueva Granada & 26 & 428 & 333 & 27 & 446 & 351 & 25 & 368 & 274 \\
\hline Universidad del Magdalena & 27 & 444 & 349 & 28 & 455 & 358 & 28 & 379 & 284 \\
\hline Universidad de Nariño & 28 & 457 & 360 & 30 & 488 & 390 & 26 & 372 & 277 \\
\hline Universidad Autónoma de Bucaramanga & 29 & 479 & 381 & 25 & 434 & 339 & 19 & 334 & 245 \\
\hline Universidad de La Salle & 30 & 509 & 410 & 32 & 516 & 416 & 35 & 415 & 315 \\
\hline
\end{tabular}




\begin{tabular}{|c|c|c|c|c|c|c|c|c|c|}
\hline Universidad de San Buenaventura & 31 & 512 & 413 & 31 & 507 & 407 & 27 & 373 & 278 \\
\hline Universidad Antonio Nariño & 32 & 534 & 432 & 33 & 537 & 434 & 30 & 391 & 295 \\
\hline Universidad Santo Tomas & 34 & 537 & 435 & 41 & 615 & 506 & 48 & 478 & 370 \\
\hline Universidad de Sucre & 33 & 535 & 433 & 35 & 560 & 454 & 36 & 439 & 333 \\
\hline Universidad Surcolombiana & 35 & 553 & 450 & 36 & 568 & 462 & 33 & 408 & 308 \\
\hline Universidad del Atlántico & 36 & 559 & 455 & 39 & 609 & 500 & 37 & 442 & 336 \\
\hline Fundación Universitaria San Martin & 37 & 614 & 504 & 40 & 614 & 505 & 38 & 446 & 340 \\
\hline Escuela de Ingeniería de Antioquia & 38 & 628 & 517 & 46 & 637 & 525 & 43 & 463 & 357 \\
\hline Instituto Tecnológico Metropolitano & 39 & 644 & 532 & 58 & 745 & 628 & 66 & 541 & 431 \\
\hline Universidad Católica de Colombia & 40 & 645 & 533 & 45 & 628 & 519 & 50 & 480 & 372 \\
\hline Universidad Popular del Cesar & 41 & 648 & 536 & 42 & 616 & 507 & 34 & 411 & 311 \\
\hline Universidad Santiago de Cali & 42 & 653 & 541 & 47 & 642 & 530 & 41 & 454 & 348 \\
\hline Universidad Autónoma de Manizales & 43 & 659 & 547 & 43 & 622 & 513 & 40 & 449 & 343 \\
\hline Politécnico Colombiano Jaime Isaza Cadavid & 44 & 668 & 554 & 49 & 654 & 540 & 45 & 472 & 364 \\
\hline Universidad de Santander & 45 & 679 & 565 & 51 & 680 & 565 & 55 & 489 & 380 \\
\hline Universidad de Manizales & 46 & 689 & 575 & 54 & 707 & 590 & 49 & 479 & 371 \\
\hline Escuela Colombiana de Ingeniería Julio Garavito & 47 & 693 & 578 & 52 & 700 & 583 & 54 & 488 & 379 \\
\hline Universidad Pedagógica Nacional, Bogotá & 48 & 706 & 591 & 60 & 766 & 647 & 60 & 512 & 403 \\
\hline Fundación Universitaria de Ciencias de la Salud & 49 & 711 & 596 & 55 & 714 & 597 & 44 & 471 & 363 \\
\hline Universidad Central & 50 & 712 & 597 & 63 & 795 & 675 & NA & NA & NA \\
\hline
\end{tabular}

COL: Colombia; IBE: Iberoamérica; LAC: Latino América.

Al analizar el factor relacionado con la producción científica (PC), la Universidad Nacional de Colombia ocupa el primer lugar con 4.302 publicaciones, seguido de la Universidad de Antioquia con 2.326, en tercer lugar la Universidad de los Andes con 1.709. Existe una diferencia significativa $(p \leq 0.05)$ en este factor con relación a las publicaciones entre las cinco primeras universidades. Al comparar las tres primeras universidades internacionales del ranking: Universidad de Sao Paulo con 44610, Universidad Nacional Autónoma de México con 18350, Universidade Estadual de Campinas con 16154; se nota una amplia diferencia de este factor con las cinco primeras universidades colombianas.

En la figura 2 se describe el factor de colaboración internacional, relacionado con las publicaciones científicas en donde uno o más coautores pertenecen a diferentes países; se observó un aumento en todas las universidades del país. Las tres primeras universidades colombianas que sobresalen con este indicador son: Universidad de Pamplona (68.7), Universidad del Atlántico (63.3) y Universidad Jorge Tadeo Lozano de Bogotá (63.1), las cuales tienen una clasificación dentro del Ranking nacional de 23, 36, 25, respectivamente. Este factor es importante por la visibilidad; sin embargo, al parecer no pondera lo suficiente en la producción científica de la universidad ni en el puesto final en el ranking. Al comparar el factor de colaboración internacional de las tres primeras universidades latinoamericanas como son Universidad de Chile
(43.9), Universidad de Buenos Aires (39.8) y Universidad Nacional Autónoma de México (38.3), las universidades colombianas paradójicamente mostraron una mejor cooperación internacional; es decir, que a pesar del mayor desarrollo que tienen las tres universidades extranjeras mencionadas, las colombianas repuntaron con este factor.

También se evaluó la calidad científica promedio (CCP), la cual mide las citaciones de las universidades, que es una especie de factor de impacto universitario; una puntuación de 0.8 demuestra que una universidad es citada un $20 \%$ menos que la media mundial y un valor de 1.3 indica que es citada un $30 \%$ más que la media mundial. Este valor en general para todas las instituciones del país está muy por debajo de la puntuación media mundial, las tres universidades que sobresalieron fueron: Universidad Antonio Nariño con una puntuación de 2.5, Universidad El Bosque con 1.2 y las Universidades de Caldas, Escuela Colombiana de Ingeniería Julio Garavito y de los Andes, con puntuación de 1 (Figura 3 ). El resto de universidades no superaron una puntuación de 0.9 con excepción de las Universidades de Caldas y de los Andes puestos 12 y 3 respectivamente; no se observó relación con la ubicación en el ranking. Al comparar las universidades latinoamericanas que ocupan los tres primeros puestos en este factor de medición ninguna superó la puntuación de 1 . Es importante que las IES de Colombia se enfoquen en las publicaciones de calidad e impacto para mejorar el peso y la visibilidad internacional. 


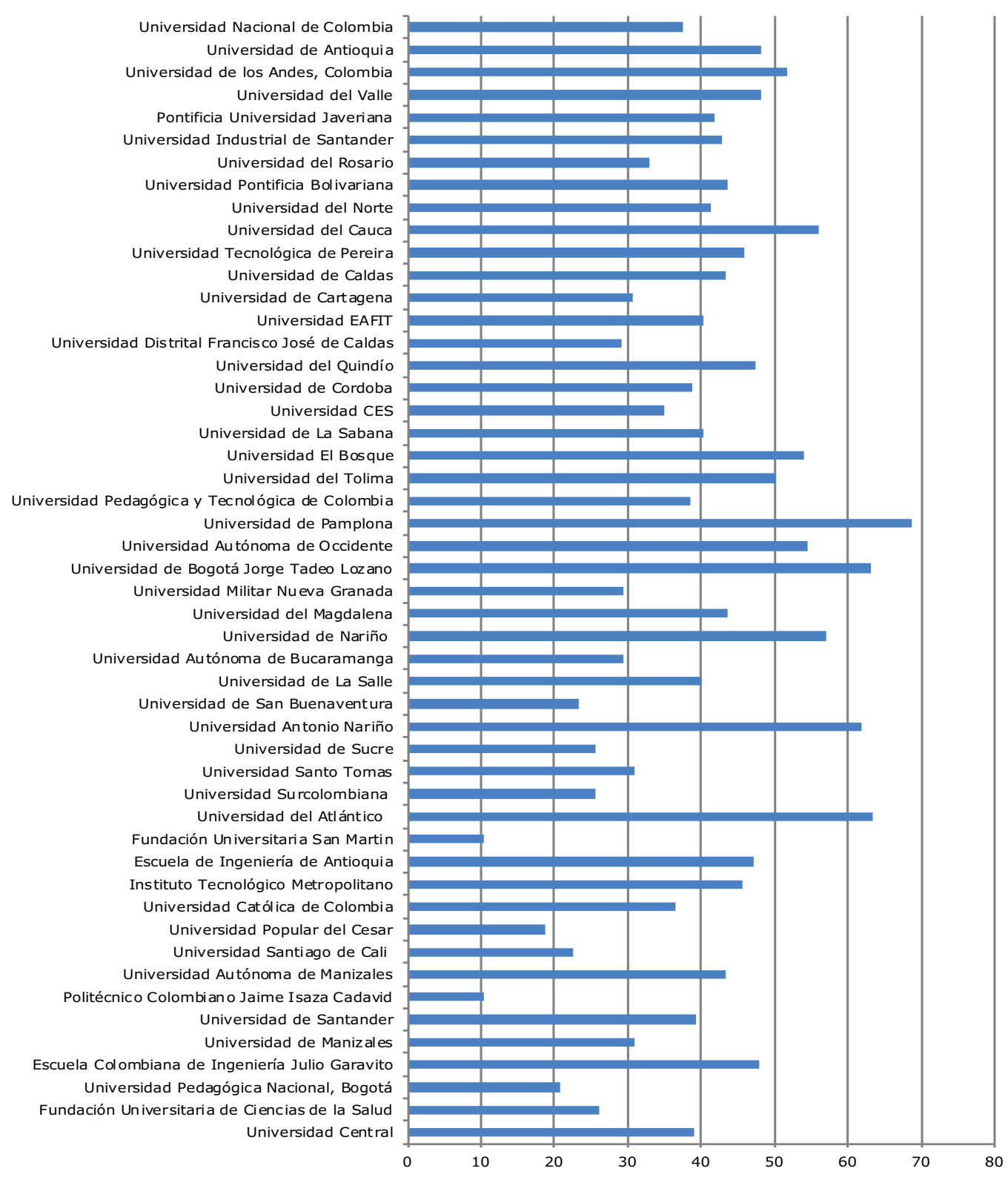

Figura 2. Relación de Colaboración Internacional en publicaciones con autores de diferentes nacionalidades.

SCImago incluyó el factor del porcentaje de publicaciones de las universidades en revistas del primer cuartil SJR. Esto mide la influencia o prestigio científico de las revistas mediante un análisis de cantidad y procedencia de las citas que recibe una revista científica (factor de impacto). Sólo 14 universidades de las estudiadas se mantuvieron sobre el promedio anteriormente mencionado y de acuerdo con este análisis las tres mejores IES colombianas fueron: Universidad del Bosque (46.5), Escuela de Ingeniería de Antioquia (38.9) y Universidad de Pamplona (38.8). Al comparar esta medición con las tres primeras universidades Latinoamericanas: Universidad de Buenos Aires (51.5), Universidad Nacional Autónoma de México (44.9), Universidad de Chile (42.9) se observa una mayor puntuación en las 3 latinoamericanas.

Finalmente, el ratio de excelencia, mide el porcentaje de la producción (output) de las instituciones nacionales, lo cual indica que una universidad se encuentra incluida en el $10 \%$ de los trabajos más citados del mundo en sus respectivos campos científicos. Esto es muy importante y demuestra el esfuerzo de las universidades en publicar sus investigaciones en revistas de calidad. Las tres universidades 


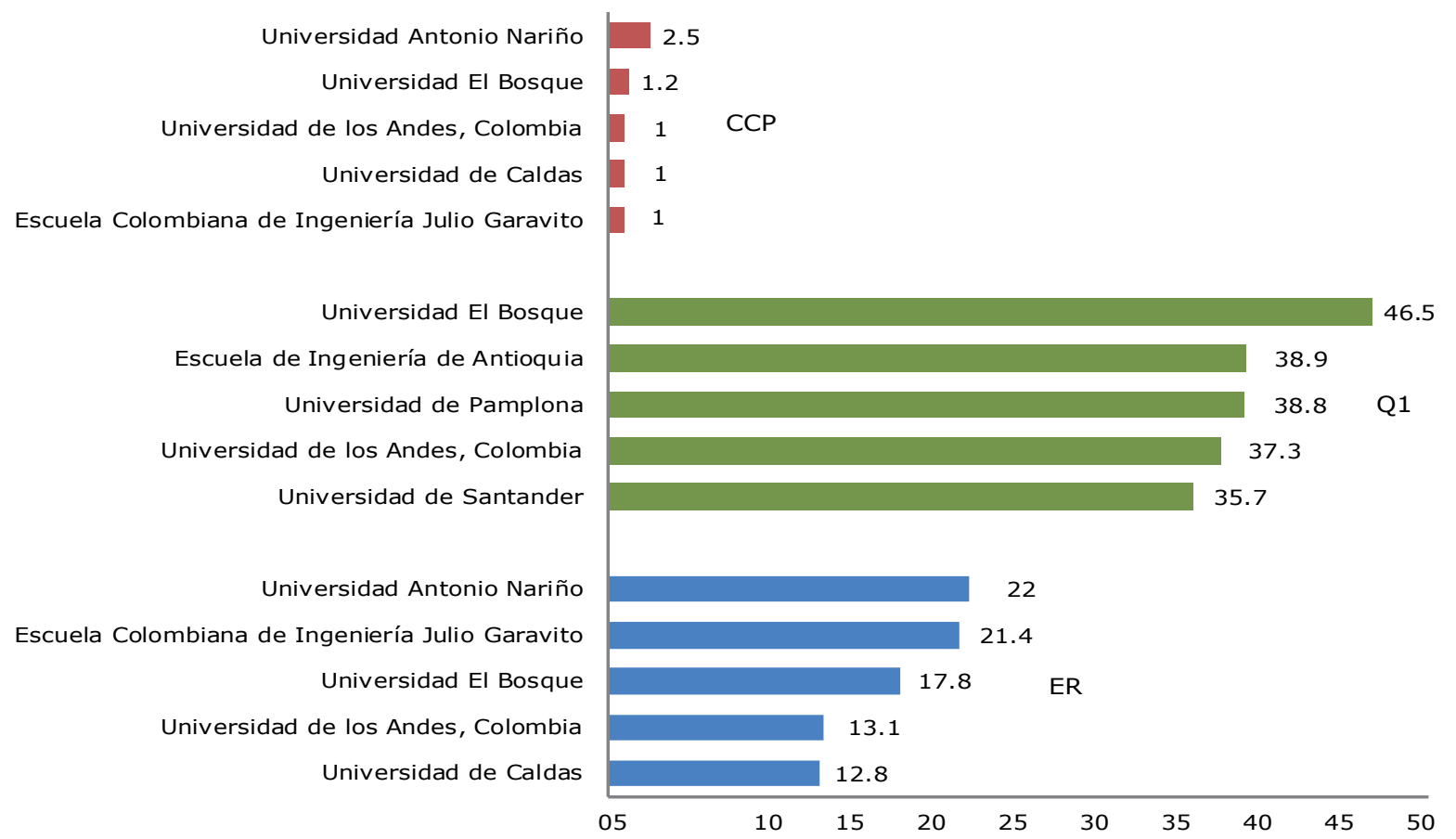

Figura 3. Distribución de las Universidades según su Calidad Científica Promedio (CCP), Publicaciones en el primer cuartil (Q1) y Ratio de Excelencia (ER).

que se destacaron en esta categoría fueron: Universidad Antonio Nariño, Escuela Colombiana de Ingeniería Julio Garavito y Universidad El Bosque (Figura 3).

\section{DISCUSIÓN}

La mayoría de las universidades que se destacan en los primeros puestos del ranking, le han apostado a la investigación como la variable crucial que "arrastra" por si sola a los otros factores. Esto significa tener mejores profesores, ojalá la mayoría con doctorado y maestria, que en últimas son los que impulsan las investigaciones de calidad que generan publicaciones. El mundo académico exige tambien la unión de grupos de investigación, en ese sentido el factor de la cooperación internacional es importante para tener un puesto de honor en la clasificacion de las IES.

El $44 \%$ de las universidades en el ranking son públicas, de ellas las 2 primeras han liderado durante los 3 últimos años la clasificación (Tabla 1). Entre las 10 primeras del pais la mitad son públicas, lo que es relevante, ya que indica que los aportes del estado en estas universidades han sido bien invertidos. Esta inversión también se ve reflejada con las publicaciones en áreas específicas en los analisis bibliométricos previos, donde las IES han aportado más del $85 \%$ de las publicaciones (11).
Aunque las carreras de pregrado son muy importantes para la nación, las 50 primeras IES de Colombia (Tabla 1) le han apostado fuerte a las escuelas de postgrado como generadores de nuevos conocimientos. Es claro que los estudiantes de postgrado (Maestría y Doctorado), son los que inciden en la clasificación de las universidades, ya que ellos son quienes generan la mayoría de las publicaciones con sus tutores. Las IES internacionales como la universidad de Sao Pablo (Brasil), por ejemplo, primera en el escalafón de SCImago (Tabla 1), el $60 \%$ de sus estudiantes están matriculados en escuelas de postgrados. Esto requiere repensar si las universidades de Colombia deben aumentar una cobertura anodina con estudiantes de pregrado o apostarle a este factor al menos por un decenio. Esto permitira subir en el ranking sin desconocer desde luego, el papel de la universidad en la preparación de jovenes profesionales que requiere el país, así como el relevo generacional que ellos representan.

Con relación a la producción científica, se puede observar claramente un incremento en la mayoría de las instituciones de educación superior en la búsqueda de divulgar la información que se desarrolla en cada una de ellas. Esto se refleja en el creciente número de revistas científicas que cada año hacen parte de la clasificación nacional de PUBLINDEX COLCIENCIAS $(12,13)$, esta última ha motivado y exigido el fortalecimiento 
a las revistas nacionales con el objeto de acceder a las bases de datos internacionales más importantes que son las que en última instancia muestran la visibilidad global.

Con respecto a la cooperación internacional, se debe en gran medida a la relación personal entre los investigadores del país con sus colegas de universidades extranjeras, pero no porque en Colombia exista una politica que invierta en ese factor. La cooperacion internacional es una excelente estrategia para las universidades, dado que al publicar con instituciones mejor escalafonadas su visibilidad aumenta y de paso su reconocimeinto de trabajo en grupo, además de todos los créditos académicos que esta relación implica en la generación del nuevo conocimiento.

Al observar la calidad científica promedio, se ve una disminución en las citaciones de las instituciones del país. Este factor afecta a las IES en Colombia, dado por el menor número de revistas científicas del país en bases de datos de más alto prestigio como son ISI o SCOPUS, entre otras, lo que reduce el número de las citaciones a instituciones colombianas.

El porcentaje de publicaciones en revistas del primer cuartil SJR, se torna muy reducido y mucho se debe al incremento de las exigencias que tienen las revistas con mayor factor de impacto, por lo cual se deben incrementar políticas de calidad científica de las publicaciones que se generan dentro de las instituciones educativas. Estas políticas en términos generales están promulgadas por COLCIENCIAS-PUBLINDEX y es conveniente que las IES colombianas las tengan muy en cuenta a la hora de financiar las revistas institucionales.

Si bien es cierto que las universidades públicas ocupan los dos primeros lugares del ranking desde el 2010, es importante resaltar que algunas universidades privadas como Antonio Nariño, el Bosque y la Escuela Colombiana de Ingenieria, a pesar de no estar en los 15 primeros puestos del pais, han hecho esfuerzos destacados para mejorar los indicadores de calidad científica, realizando publicaciones en el primer cuartil y radio de excelencia. Las universidades públicas de Caldas (calidad cientifica) y Pamplona (primer cuartil) se muestran interesadas en mejorar esos indicadores, política y estrategia que deberían seguir todas las universidades colombianas. Es muy probable que en la proxima clasificación este esfuerzo se vea reflejado en las posiciones del ranking.

En conclusión, cada día se le da mayor importancia a las clasificaciones o rankings que se desarrollan a nivel mundial, aunque varía en la forma en que cada país asume las responsabilidades y estrategias relacionadas con este tipo de categorizaciones. Las IES de Colombia deben mandatoriamente apostarle a la calidad vista al menos desde la perspectiva de los cinco factores analizados por SCImago. Aunque los rankings no obligan a las universidades a aceptar los escalafones; el ignorarlos no significa otra cosa que un autoaislamiento en un mundo cada día más globalizado.

\section{REFERENCIAS}

1. Miñana CB, Gregorio RJ. Calidad, evaluación y acreditación en la educación superior: a propósito de un Proyecto de reforma en Colombia. Pensamiento Jurídico 2011; (31): 155-185.

2. Aquevedo E. Ranking (2012, Shanghai) de las 500 mejores universidades del mundo. [En linea]. 2012. [Accesado octubre de 2012]. URL Disponible en: http://aquevedo. wordpress.com/2012/08/17/rankingshanghai-2012-de-mejores-universidadesdel-mundo/

3. Fayad R. Anotaciones para una reflexión sobre la educación superior en Colombia. Rev Fac Med 2010; 18(1):123-133.
4. Docampo D. "Rankings internacionales y calidad institucional". Revista de Educación 2008; Número Extraordinario:149-176. URL Disponible en: http://www. revistaeducacion.mec.es/re2008/ re2008_07.pdf

5. Martínez RF. Los rankings de universidades: una visión crítica. Rev Educ Sup 2011; 40(157):77-97. URL Disponible en: http:// scielo.unam.mx/pdf/resu/v40n157/ v40n157a4.pdf

6. ARGU. About Academic Ranking of World Universities. [en línea]. China: Shanghai Jiao Tong University; 2013 [fecha de acceso enero de 2013]. URL Disponible en: http:// www.shanghairanking.com/aboutarwu.html 
7. The World University Rankings. [en línea]. England: Times Higher Education; 2013. [fecha de acceso enero de 2013]. URL Disponible en: http://www. timeshighereducation.co.uk/worlduniversity-rankings/

8. Ranking Mundial de Universidades en la Web. [en línea]. España: Consejo Superior de Investigaciones Científicas, CSIC. 2013. [fecha de acceso enero de 2013]. URL Disponible en: http://www.webometrics. info/

9. SJC. SCImago Journal \& Country Rank. [en línea]. España: SCImago Lab; 2013. [fecha de acceso enero de 2013]. URL Disponible en: http://www.scimagojr.com/

10. SCImago Institutions Rankings (SIR). [en línea]. España: SCImago Research Group; 2013. [fecha de acceso enero de 2013]. URL Disponible en: http://www.scimagoir.com
11. Ríos R, Mattar S, González M. Análisis bibliométrico de las publicaciones sobre enfermedades infecciosas en Colombia, 2000-2009. Rev Salud Pública 2011; 13(2):298-307.

12. González M, Mattar S. Índice Bibliográfico Nacional - IBN Publindex 2004 - 2010. Rev MVZ Córdoba 2010; editorial 15(3): 2145-2146.

13. Indicadores Publindex 2002-2011. [en línea]. Colombia: Publindex, Colciencias; 2011. URL Disponible en: http://www. colciencias.gov.co/sites/default/files/ ckeditor_files/files/INDICADORES $\% 20$ GENERALES\%2OPUBLINDEX\%202011.pdf. 